\title{
Why statins are beneficial
}

New research demonstrates that men who take statins at the time of starting androgen deprivation therapy (ADT) for the treatment of hormone-sensitive prostate cancer have an extended time to disease progression. The report in JAMA Oncology also describes that statins compete with dehydroepiandrosterone sulfate (DHEAS) for uptake into prostate cancer cell lines in vitro, presenting a possible mechanism underlying the clinical findings.

The testosterone precursor DHEAS can be taken up by prostate cancer cells via a receptor expressed from the SLCO2B1 gene. "We have been studying the SLCO2B1-expressed transporter for several years and think it is central to androgen metabolism," explains Philip Kantoff from the Dana-Farber Cancer Institute in Boston, USA, senior author of the study. "When we learned that statins also use this transporter, we decided to look at the interaction between statins and androgen metabolism and response to androgen depletion."

In in vitro experiments in one androgen-dependent and one partially androgen-dependent cell line, the team tested the ability of four statins to decrease the uptake of DHEAS at physiological levels into the cells. The statins competed with DHEAS for cellular uptake with varying degrees of efficiency in a cell-line-dependent manner. Knockdown of expression of the SLCO2B1-encoded receptor limited DHEAS uptake by $>50 \%$, corroborating the importance of the receptor in DHEAS uptake. In addition, incubation of cells with atorvastatin at a level that would be found in patient serum inhibited DHEAS-induced cell proliferation.

The investigators also evaluated the clinical effect of statin use on disease progression retrospectively in 926 men with hormone-sensitive prostate cancer with or without metastases. $31 \%$ of patients were taking statins when they commenced ADT. At diagnosis, men taking statins were more likely to have lowerstage disease and they were less likely to have nodal involvement or de novo metastases than men not using statins.

At analysis at a median follow-up period of 5.8 years, $70 \%$ of men had experienced disease progression according to PSA level. Importantly, men who were taking statins at the start of ADT had a significantly longer time to progression (27.5 months versus 17.4 months, $P<0.001$ ). After adjusting for predefined prognostic factors, including Gleason score or the presence of metastases, the relative risk reduction for disease progression was $17 \%$ (95\% CI 0.69-0.99).

Several trials are underway to prospectively study the effect of statins on prostate cancer and will further clarify the role of these agents as adjuvants in prostate cancer treatment.

\section{Clemens Thoma}

Original article Harshman, L. C. et al. Statin use at the time of initiation of androgen deprivation therapy and time to progression in patients with hormone-sensitive prostate cancer. JAMA Oncol. doi:10.1001/ jamaoncol.2015.0829 